\section{Teste de uma metodologia para avaliar a organização, acesso e qualidade técnica do cuidado na atenção à diarréia na infância}

\author{
Testing a methodology to evaluate \\ organization, access, and technical quality \\ of care for childhood diarrhea
}

Rosana Machado Lopes 1

Lígia Maria Vieira-da-Silva 1

Zulmira M. de Araújo Hartz 2,3

\footnotetext{
1 Instituto de Saúde Coletiva Universidade Federal da Bahia, Salvador, Brasil. 2 Escola Nacional de Saúde Pública, Fundação Oswaldo Cruz, Rio de Janeiro, Brasil.

3 Faculté de Médecine, Université de Montréal, Montréal, Canada.

Correspondência R. M. Lopes

Instituto de Saúde Coletiva, Universidade Federal da Bahia. Rua Padre Feijó 29, 4 o andar, Salvador, $B A$ 40110-170, Brasil. rmachado@ufba.br
}

\begin{abstract}
In order to evaluate the issues of access and technical quality in actions to control diarrhea in a selected municipality in the State of Bahia, Brazil, a study of multiple cases was conducted with two levels of analysis: practices themselves and the coordination between them. Cases were defined as hospitalization (8) or death (3) due to diarrhea. All deaths were investigated, and hospitalizations were submitted to intentional selection. Aspects and approaches vis-à-vis technical quality evaluation and estimation of the degree of implementation in diarrhea control measures were derived from the theoretical model. Health care trajectories for diarrhea were reconstituted from interviews and patient chart data. The results identified: organizational barriers related to long waiting time at primary care units; geographic barriers, or the distance from users' place of residence to health services; and financial barriers due to shortage of financial resources for transportation. Quality of care was considered inadequate, and diarrhea control measures were classified as insufficient in practically all of the dimensions analyzed.
\end{abstract}

Health Services Evaluation; Diarrhea; Health Services Access; Quality of Health Care

\section{Introdução}

Nas últimas quatro décadas, tem-se observado uma tendência de queda na taxa de mortalidade infantil (TMI), tanto em países desenvolvidos, quanto naqueles em desenvolvimento 1 . No Brasil, verificou-se redução desse indicador de 115, em 1960, para 36 óbitos por mil nascidos vivos, em 1998 2. Segundo os resultados preliminares do censo demográfico de 2000, o indicador ainda permanece elevado, visto que apresenta uma TMI estimada em 28,6 óbitos por mil nascidos vivos em 20013.

O país apresenta ainda diferenças entre indicadores de mortalidade quando se comparam as suas regiões 3,4. Essa distribuição desigual entre as regiões reflete, em parte, as diferentes condições de vida da população. As intervenções sanitárias vêm sendo responsabilizadas pelo declínio em $33,0 \%$ nas taxas de mortalidade infantil no Brasil, entre as décadas de 80 e 90, devido ao fato de não se ter observado, naquele período, alteração no modelo de desenvolvimento econômico, caracterizado, historicamente, pela distribuição desigual de renda 5 . Um estudo mais recente aponta evidências no sentido de indicar a contribuição da queda de fecundidade para essa redução da mortalidade infantil, principalmente, na década de 806 .

Em Salvador, Bahia, entre 1980 e 1998, o coeficiente de mortalidade infantil (CMI) por diarréia reduziu-se em $91,4 \%$. Foram apontados al- 
guns fatores que podem ter contribuído para isso, como o aumento da escolaridade, incentivo ao aleitamento materno, implantação da terapia de reidratação oral e uma relativa expansão dos domicílios ligados à rede geral de abastecimento de água 1.

Vale salientar um estudo que analisou a evolução da mortalidade infantil por causas evitáveis naquele município, no período compreendido entre 1980 a 1998. Tal estudo classificou os óbitos por diarréia como uma das principais causas do grupo de doenças parcialmente redutíveis por diagnóstico e tratamento precoces, além de revelar que esses óbitos foram os que apresentaram maior redução na taxa de mortalidade infantil naquele período 7 .

Apesar de existirem tantos recursos tecnológicos de fácil aplicabilidade, cada criança, nos países em desenvolvimento, apresenta uma média de três episódios de diarréia por ano, e, embora se tenha observado um decréscimo nas taxas de mortalidade por diarréia, esta é ainda uma das principais causas de morbi-mortalidade em crianças ${ }^{8}$. As doenças diarréicas foram a segunda causa de internação para menores de cinco anos, ocorrida na rede de saúde pública e conveniada do SUS, no Brasil, no ano de 20013 . Dessa forma, continuam constituindose em um problema de saúde pública, visto que a ocorrência de hospitalizações e/ou óbitos infantis revelam falência nas ações de controle.

Os estudos revisados sobre a mortalidade infantil têm descrito a magnitude, a evolução, os fatores de risco ambientais e os determinantes sócio-econômicos desse agravo. Poucos trabalhos têm buscado investigar acerca dos problemas relacionados aos sistemas de saúde. Além disso, a eficácia das ações dirigidas à população infantil tem variado em diferentes contextos, fato que impõe a investigação destes 9 .

Levando em consideração a escassez de estudos que se proponham a investigar os problemas dos serviços de saúde relacionados a casos de insucesso no controle das diarréias, o presente trabalho pretende contribuir para responder à seguinte pergunta: por que algumas crianças ainda morrem e são internadas por diarréia? Coerente com essa questão, a presente investigação teve como objetivos avaliar a qualidade técnico-científica do cuidado e a acessibilidade ao sistema de serviços de saúde das crianças menores de quatro anos que foram a óbito e/ou internadas com diagnóstico de diarréia e estimar o grau de implantação das ações relacionadas ao controle da diarréia, em um município selecionado do Estado da Bahia.

Busca-se, assim, entender em que medida a organização do sistema e as práticas assisten- ciais adotadas pelos profissionais de saúde estão atuando com eficácia para a prevenção das diarréias na infância, bem como o cuidado delas. Pretende-se, também, contribuir com o gestor municipal no desenvolvimento de técnicas para a avaliação do sistema de saúde local, na medida em que a diarréia pode ser utilizada como condição traçadora e as internações e óbitos causados por ela, como eventos-sentinela.

\section{Metodologia}

\section{Referenciais teóricos}

\section{- Modelo teórico}

Foi desenvolvido um modelo teórico adaptado de Paim 10,11, buscando situar as ações para o enfrentamento da diarréia infantil em relação aos diferentes níveis de controle: de causas, riscos e danos, na perspectiva da vigilância da saúde. A promoção à saúde foi selecionada como ação de controle de causas e os atributos de acessibilidade, qualidade técnica e integralidade do cuidado como relacionados ao controle de danos (Figura 1).

O conceito de modelos assistenciais adotado neste estudo é aquele que os considera como combinações tecnológicas destinadas ao enfrentamento de problemas de saúde individuais e coletivos, em determinados espaçospopulação, incluindo ações sobre o ambiente, grupos populacionais, equipamentos comunitários e usuários de serviços de saúde. E que deve priorizar o controle de causas e riscos sem descuidar do controle de danos, mediante uma oferta predominantemente organizada de serviços de saúde 10,11. Contudo, as intervenções baseadas nas medidas de promoção da saúde se aplicam à melhoria das condições e dos estilos de vida de grupos populacionais específicos, apoiando-se, amplamente, em atividades de educação e comunicação em saúde e na formulação de "políticas públicas saudáveis" nas várias esferas de governo, inclusive, na municipal (Ferraz, 1993, 1998, apud Paim 12).

\section{- O uso de traçadores \\ e eventos-sentinela}

A seleção de condições que podem ser consideradas como traçadoras apresenta-se como uma alternativa para abordar problemas complexos do sistema, como a avaliação da qualidade da assistência, podendo gerar dados de fácil compreensão, capazes de retroalimentar o sistema de prestação de serviços de saúde 13. Já 
Controle de causas

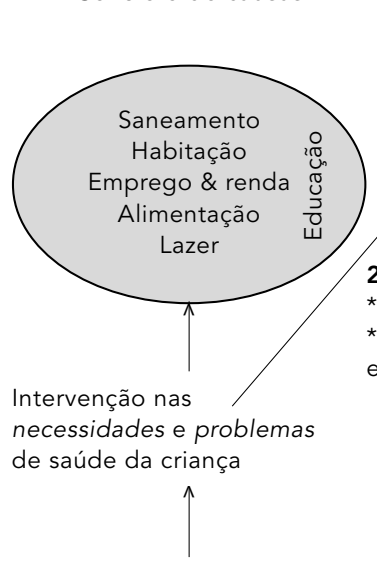

Políticas públicas transetoriais

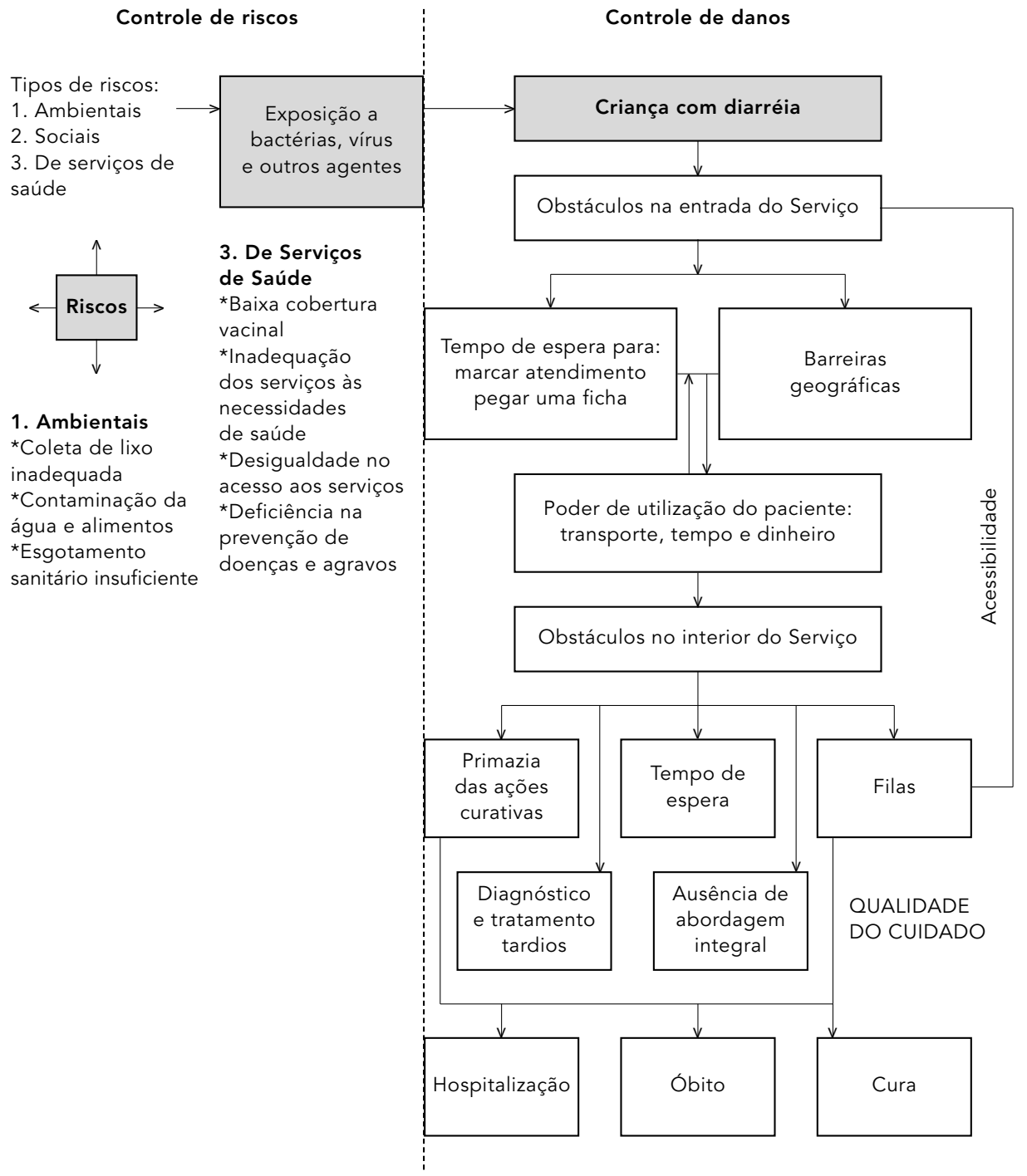

Fonte: Lopes 21. Adaptado de Paim 10,11.

eventos-sentinela são aqueles que correspondem à ocorrência de uma situação indesejável, que pode acontecer em decorrência de uma falha no produto da atenção à saúde, chegando a ocasionar uma morte desnecessária, doença ou invalidez 14 . Os eventos-sentinela podem ser usados para a avaliação de serviços de saúde, sendo úteis para elucidar nós críticos existentes na assistência e no funcionamento dos serviços. Esses dois conceitos acima tratados foram utilizados para o desenvolvimento da estratégia metodológica deste estudo, já que a diarréia foi considerada como uma condição traçadora e as internações e os óbitos infantis foram considerados eventos-sentinela.

\section{- Acessibilidade, qualidade técnica e integralidade}

A avaliação do funcionamento dos serviços de saúde será feita com base na análise da acessibilidade, da qualidade técnica do cuidado e do grau de implantação das ações relacionadas ao controle da diarréia no município estudado. A acessibilidade será aqui compreendida como a relação entre o conjunto de obstáculos na bus- 
ca da atenção à saúde e a capacidade da população para superá-los 15 , cujo desfecho pode interferir negativamente no manejo do problema 16 .

A qualidade técnico-científica do cuidado de saúde, segundo Vuori 17, é a aplicação apropriada do conhecimento médico disponível, bem como da tecnologia, no cuidado com o paciente. Para Donabedian 18, essa avaliação está voltada para a análise do processo, pois engloba as atividades ou os procedimentos empregados pelos profissionais de saúde para transformar os recursos em resultados. Isto é, a análise processual está direcionada a identificar os procedimentos "necessários" para o manejo dos casos atendidos e verificar se eles realmente foram aplicados apropriadamente 19 . Nesta pesquisa, o tratamento médico instituído para a diarréia será foco de investigação, uma vez que poderá nos revelar a qualidade da assistência prestada para as crianças que foram hospitalizadas e/ou a óbito por esse agravo.

Apesar de o conceito de integralidade do cuidado ser usado em vários sentidos, utilizaremos aquele que considera a integralidade relacionada, principalmente, com as ações de promoção e prevenção à saúde 20 . Seguindo essa tendência, Paim 10,11 propõe o modelo de vigilância da saúde, apontando que as intervenções na atenção à saúde deverão ter como eixo de prioridade as ações de controle de riscos e causas.

\section{Desenho do estudo}

Realizou-se um estudo de casos múltiplos com dois níveis de análise, os quais estavam aninhados em um estudo de caso municipal, como parte de um projeto mais amplo denominado Avaliação da Descentralização da Saúde na Bahia - do qual a presente investigação faz parte (Vieira-da-Silva et al., 2002, apud Lopes 21). O referido estudo articulou duas estratégias metodológicas complementares: (a) um estudo de séries temporais de amostra probabilística estratificada segundo condições de vida de 74 municípios baianos, onde quatro condições traçadoras, como hipertensão, tuberculose, infecções respiratórias agudas e a diarréia, foram investigadas; (b) uma análise de implantação das estratégias e programas relacionados com a descentralização da gestão da saúde em cinco municípios baianos na gestão plena do sistema. O presente estudo teve por objetivo analisar em profundidade um desses municípios, que se encontrava em estágio intermediário da descentralização, e apenas um dentre os quatro traçadores investigados - a diarréia -, a fim de indagar as razões da persistência de óbitos evitáveis por condições tão banais, em municípios já em estágio intermediário da descentralização e que vinham controlando com sucesso esse agravo. Esse município, denominado no projeto original de "A", assumiu a gestão plena do sistema de saúde em 1999, e sua rede de serviços de saúde públicos, em 2001, contava com mais de dez unidades de saúde da família, cerca de cinco unidades de atenção básica tradicionais, cinco policlínicas, duas unidades móveis e um hospital estadual 21. Por razões éticas, o nome do município foi omitido, bem como características que o possam identificar foram evitadas ou informadas com medidas aproximadas.

\section{Definição e seleção dos casos}

Cada óbito e cada internação por diarréia foram considerados como caso e foram selecionados conforme os seguintes critérios, para o ano de 2001: (a) analisaram-se todos os óbitos ocorridos entre o 29o dia de vida e um ano de idade da criança, por local de residência; (b) estudaram-se todas as hospitalizações ocorridas no mês de outubro, o que correspondeu a $30 \%$ daquelas por diarréia, com período de permanência superior a 24 horas e em crianças menores de quatro anos. Em ambos os casos, as crianças deveriam ter apresentado, pelo menos, uma das seguintes causas básicas para a internação e/ou óbito: códigos 008-009 e A00A09, das 9a e 10a Revisões da Classificação Internacional de Doenças/CID, respectivamente 22,23. O total de casos correspondeu a três óbitos e nove internamentos de crianças. Foram analisadas apenas nove trajetórias, pois, em dois casos de óbito, as crianças também haviam sido hospitalizadas; um caso de internamento foi descartado em virtude da não-localização do prontuário ambulatorial, do desconhecimento do agente comunitário de saúde sobre o caso e da não-identificação do domicílio da criança.

\section{Níveis de análise, dimensões, subdimensões e critérios \\ - Qualidade técnico-científica do cuidado ambulatorial}

Elaborou-se um instrumento para avaliação da qualidade técnico-científica do cuidado ambulatorial 21, com base nas recomendações do Ministério da Saúde para a Atenção Integrada às Doenças Prevalentes na Infância (AIDPI), uma vez que essa estratégia se apresenta como um novo enfoque de abordagem à saúde da 
criança no primeiro nível de assistência, integrando ações curativas com medidas de prevenção e promoção da saúde ${ }^{3}$.

Para tanto, foi definida uma relação de critérios, baseada no estudo de Araújo 24 e na estratégia AIDPI. Os critérios inicialmente selecionados e que constam na ficha específica foram submetidos, previamente, a um painel de especialistas, composto por três médicos pediatras, dois professores da Faculdade de Medicina da Universidade Federal da Bahia e uma técnica na área de saúde da criança da Secretaria Estadual de Saúde da Bahia. Foi utilizada a técnica Delphi simplificada com duas rodadas, para o refinamento desse instrumento 25 , e, após um parecer individual dos três componentes, chegou-se a um consenso quanto aos critérios que deveriam permanecer para avaliar os prontuários ambulatoriais. As sugestões do painel de especialistas foram acatadas e outras modificações foram incorporadas para melhorar o instrumento supracitado.

A classificação utilizada para a avaliação da adequação do cuidado tomou como referência aquela elaborada por Araújo 24, porém foi adaptada para o agravo em estudo - as afecções diarréicas; considerou-se, também, a classificação proposta pelo painel de especialistas.

Foram definidos os seguintes componentes para a avaliação da qualidade técnica do cuidado (QTC), que constam da ficha: (a) anamnese; (b) exame físico; (c) diagnóstico; (d) tratamento; (e) conclusão do caso. Para cada um desses componentes, foram selecionados critérios para avaliação (Tabela 1).

\section{- Avaliação do grau de implantação das ações de controle da diarréia}

Para avaliação do grau de implantação das ações de controle da diarréia no município estudado, foi elaborada uma matriz composta por dois níveis de análise. O primeiro deles diz respeito às práticas assistenciais, e o segundo refere-se à coordenação destas, a qual compreende a integração de todos os serviços relacionados à saúde, onde quer que tenham sido recebidos 26. Para viabilizar essa coordenação, faz-se necessário que a rede de serviços de saúde esteja regionalizada, a atenção primária seja a porta de entrada para o sistema e a transferência de informações seja garantida através de um sistema de referência e contra-referência eficiente.

Essa matriz foi subdividida em dimensões, subdimensões e critérios, que são os aspectos dos fenômenos estudados e foram selecionados a partir do modelo teórico. Quanto ao grau de implantação, as práticas assistenciais e a coordenação destas foram classificadas em incipientes, intermediárias e avançadas, comparando-se o observado a uma imagem-objetivo 21, definida segundo o modelo teórico.

\section{Fontes de dados e técnicas de coleta}

As informações sobre a organização do sistema de serviços de saúde do município foram retiradas do Plano Municipal de Saúde, no período de 1998 a 2001, e do Relatório de Gestão do ano de 2000.

O número de óbitos foi obtido do Sistema de Informação sobre Mortalidade, por meio do Centro de Informações de Saúde do Município e pela consulta às declarações de óbito que ainda não haviam sido digitadas no sistema de informação até a data de seis de fevereiro de 2003. Já as internações hospitalares foram, inicialmente, localizadas com o auxílio do Sistema de Informações Hospitalares do SUS (Ministério da Saúde. http://www.saude.gov.br/ tabnet.datasus.gov.br, acessado em 06/Fev/2004) e identificadas, posteriormente, na base de dados locais do hospital público estadual, por intermédio do programa de apoio à entrada de dados da Autorização de Internações Hospitalares (PAIH), versão 11.13 e apresentação do mês de outubro de 2001.

As declarações de óbito disponíveis no Centro de Informações de Saúde da Secretaria Municipal foram fotocopiadas para recuperação da informação sobre o nome da criança e da mãe, endereço e existência de hospitalização. As informações sobre as trajetórias, aqui entendidas como o percurso realizado pela criança para obter atendimento no sistema de serviços de saúde local, foram colhidas mediante entrevistas estruturadas e gravadas com: (a) a mãe ou responsável pela criança, preferencialmente aquele que acompanhou o processo de adoecimento desta; (b) o agente comunitário de saúde de referência; (c) o profissional do serviço que prestou atendimento à criança no primeiro nível de assistência e por meio da análise dos prontuários em todos os serviços de saúde, desde o menos complexo ao mais especializado.

As informações sobre as características do cuidado prestado foram obtidas nos prontuários de unidades ambulatoriais e transcritas para uma ficha específica; aquelas obtidas nos prontuários da unidade hospitalar foram transcritas para formulário equivalente aos impressos padronizados no hospital público do município. 
Tabela 1

Critérios de classificação da qualidade técnica do cuidado ambulatorial na atenção à diarréia na infância.

\begin{tabular}{|c|c|c|}
\hline \multirow[t]{2}{*}{ Critérios } & \multicolumn{2}{|l|}{ Classificação } \\
\hline & Adequado & Inadequado \\
\hline Anamnese e exame físico & $\begin{array}{l}\text { - Quando o profissional investigou para a } \\
\text { anamnese, pelo menos, a duração da diarréia } \\
\text { em dias e a presença de sangue nas fezes, } \\
\text { e quando, no exame físico, foram avaliados os } \\
\text { quatro principais sinais de desidratação: estado } \\
\text { geral, sinal da prega, olhos fundos e sede. }\end{array}$ & $\begin{array}{l}\text { - Quando o profissional não investigou todos } \\
\text { os critérios citados para ser considerado } \\
\text { adequado, tanto para a anamnese quanto para } \\
\text { o exame físico. }\end{array}$ \\
\hline $\begin{array}{l}\text { Diagnóstico: } \\
\text { Diarréia aguda, diarréia persistente } \\
\text { e disenteria sem desidratação; }\end{array}$ & $\begin{array}{l}\text { - Se o diagnóstico dado baseou-se na } \\
\text { referência afirmada ou negada dos sinais e } \\
\text { sintomas contidos na classificação, para cada } \\
\text { um dos estados diagnósticos*. }\end{array}$ & $\begin{array}{l}\text { - Se o diagnóstico dado não se baseou na } \\
\text { referência aos sinais e sintomas contidos na } \\
\text { classificação, para cada um dos estados } \\
\text { diagnósticos. }\end{array}$ \\
\hline $\begin{array}{l}\text { Diarréia aguda, diarréia persistente } \\
\text { e disenteria com algum grau de desidratação; }\end{array}$ & $\begin{array}{l}\text { *Diarréia aguda: diarréia por menos de duas sema } \\
\text { *Diarréia persistente: diarréia por } 14 \text { dias ou mais. }\end{array}$ & \\
\hline $\begin{array}{l}\text { Diarréia aguda, diarréia persistente } \\
\text { e disenteria com desidratação grave. }\end{array}$ & $\begin{array}{l}\text { *Disenteria: presença de diarréia com sangue nas } \\
\text { *Desidratação: presença de dois sinais (inquieta/ir } \\
\text { com sede, no sinal da prega a pele volta lentamen } \\
\text { *Desidratação grave: presença de dois ou mais do } \\
\text { não consegue beber ou bebe muito mal, no sinal } \\
\text { ao estado anterior). }\end{array}$ & $\begin{array}{l}\text { fezes. } \\
\text { ritada, olhos fundos, bebe avidamente } \\
\text { te ao estado anterior). } \\
\text { s sinais (letargia/inconsciência, olhos fundos, } \\
\text { da prega a pele volta muito lentamente }\end{array}$ \\
\hline Tratamento & $\begin{array}{l}\text { - Se foi prescrito de acordo com o diagnóstico } \\
\text { um antitérmico; hidratação oral com líquidos } \\
\text { (Plano A); hidratação com sais de reidratação } \\
\text { oral (Plano B); hidratação endovenosa (Plano C); } \\
\text { antibiótico para a criança com diagnóstico de } \\
\text { disenteria com comprometimento do estado } \\
\text { geral ou outro problema associado que } \\
\text { necessite de antibiótico. }\end{array}$ & $\begin{array}{l}\text { - Se o esquema terapêutico não foi compatível } \\
\text { com o diagnóstico e/ou prescrito antidiarréicos } \\
\text { e/ou antieméticos para qualquer diagnóstico } \\
\text { de diarréia } 3 .\end{array}$ \\
\hline Conclusão do caso & $\begin{array}{l}\text { - Se não tinha classificação diagnóstica grave } \\
\text { e registrou retorno para reavaliação, ou se } \\
\text { encaminhou a criança com classificação } \\
\text { diagnóstica grave para um hospital e administrou } \\
\text { alguma terapia antes de encaminhá-la (quando } \\
\text { necessário). }\end{array}$ & $\begin{array}{l}\text { - Quando a conduta não correspondeu ao } \\
\text { quadro clínico da criança. }\end{array}$ \\
\hline
\end{tabular}

\section{Plano de análise}

\section{- Para acessibilidade}

A acessibilidade foi avaliada considerando-se a descrição das trajetórias assistenciais. Estas foram reconstruídas com base nas entrevistas com a mãe, os agentes comunitários de saúde (responsáveis pela área de abrangência das mães entrevistadas), os profissionais de saúde e por intermédio das informações obtidas nos prontuários (ambulatorial e hospitalar) e na declaração de óbito. Posteriormente, foram desenhadas representações gráficas para melhor visualização das trajetórias (Figura 2).

Avaliaram-se os seguintes componentes da acessibilidade: (1) os ecológicos ou geográficos, que estão diretamente relacionados à localização das unidades de saúde na comunida- de (distância e tempo de transporte); (2) os financeiros, que se referem ao custo dos serviços (o preço que cobra o provedor para prestar os serviços de assistência à saúde) e com o transporte utilizado para o deslocamento até o serviço de saúde pretendido e (3) os organizacionais, que se originam nos modos de organização dos recursos de atenção à saúde. Estes últimos ainda podem ser classificados em: (a) obstáculos organizativos da entrada, os quais dificultam o contato inicial com o sistema (como a demora para se obter uma ficha de atendimento), e (b) obstáculos no interior de um estabelecimento de saúde (como o tempo de espera para ser atendido por um profissional de saúde) 15 .

Vale ressaltar que o paciente pode enfrentar novos obstáculos para continuar seu atendimento em outro estabelecimento de saúde, como um hospital, policlínica, laboratório, entre outros. 


\section{1ạ Trajetória:}

Policlínica pública
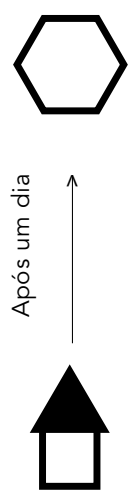

Casa
Criança 1 a 6 meses com vômito e diarréia nas últimas 24h, a mãe ofereceu soro caseiro por orientação do agente de saúde, mas seu filho não melhorou.

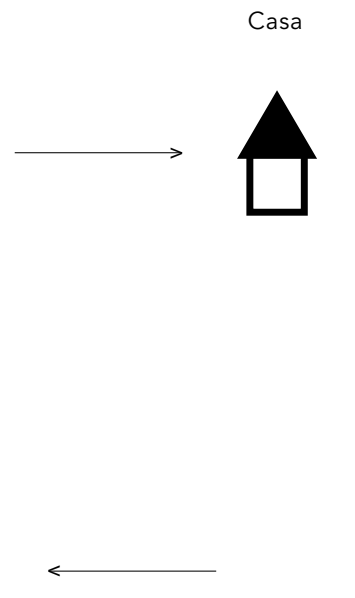

Início da doença

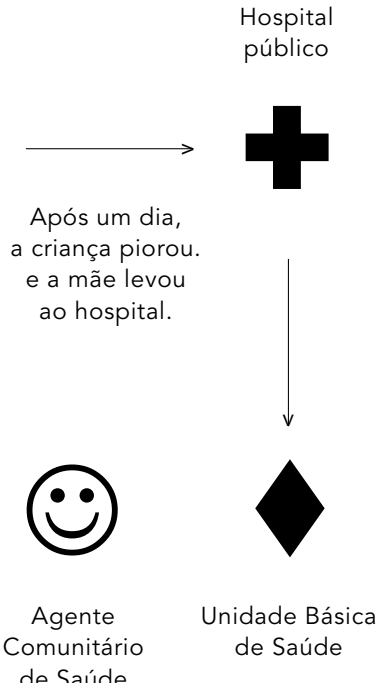

de Saúde
Após quatro dias, teve alta hospitalar e foi referido para Unidade Básica de Saúde para acompanhamento ambulatorial, mas a mãe não levou a criança porque refere que $o$ atendimento é ruim, por distribuição de fichas, e tem que acordar muito cedo para ver se consegue ser atendida.

\section{2a Trajetória:}

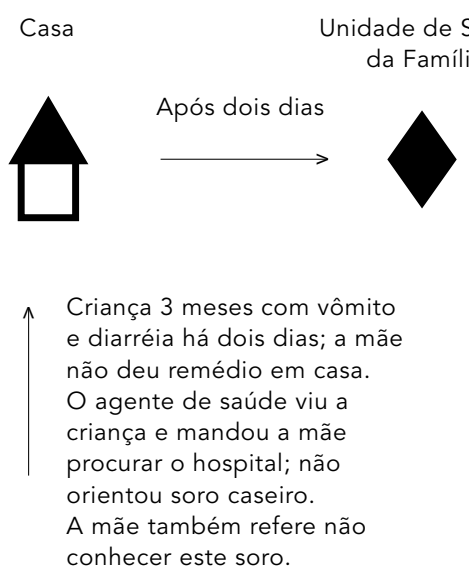

O médico atendeu a criança e orientou levar ao hospital caso piorasse o estado clínico. Não foi encontrado registro desse atendimento no prontuário da criança, nesta unidade de saúde, no período referido pela mãe.

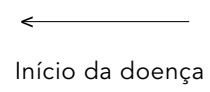

Casa

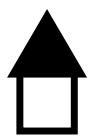

A criança piorou Comunitário de Saúde em casa.

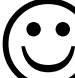

Agente

Hospital público

Após um dia
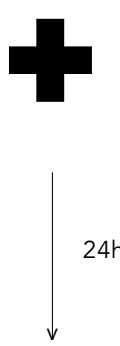

گ

Cemitério
Permaneceu internada por 24 horas e foi a óbito após parada cardiorrespiratória, porém não foi observado registro de tentativas de reanimação cardiorrespiratória. A declaração de óbito apresenta como causa básica da morte diarréia aguda, desidratação grave e insuficiência respiratória. 


\section{- Qualidade técnico-científica do cuidado}

\section{a) Análise dos prontuários ambulatoriais}

Analisou-se a qualidade técnico-científica do cuidado comparando-se o cuidado efetivamente prestado e o padrão escolhido. A informação sobre o cuidado prestado foi obtida nos prontuários. Para cada critério examinado, havia apenas uma opção de resposta, como: investigou positivo (01), que correspondeu ao critério ter sido investigado e estar presente; investigou negativo (02), que correspondeu ao critério ter sido investigado e estar ausente; não tem registro (03), que correspondeu ao critério sem anotação; ilegível (04), que correspondeu ao critério sem condições de leitura das anotações 24 .

Foi utilizada a classificação adequado (1) e inadequado (2) para os seguintes critérios: anamnese, exame físico, diagnóstico, tratamento e conclusão do caso (Tabela 1). Os prontuários ambulatoriais foram analisados por meio de critérios explícitos, preestabelecidos pelo painel de especialistas e avaliados pela pesquisadora principal.

\section{b) Análise dos prontuários hospitalares}

Os prontuários hospitalares foram distribuídos entre os membros do painel de especialistas, sendo, posteriormente, analisados individualmente por estes mediante critérios implícitos; gerou-se um parecer sobre a adequação ou não da qualidade técnico-científica de cada prontuário. Em ambos os casos, tanto para os prontuários ambulatoriais, quanto para os hospitalares, os dados foram consolidados em tabela simples do Word.

\section{- Apreciação do grau de implantação das ações de controle da diarréia}

Atribuiu-se uma pontuação para cada dimensão, subdimensão e respectivos critérios, levando em consideração as relações hierárquicas entre os fatores que potencialmente contribuem para a determinação da diarréia (Tabela 2). Essa atribuição de pesos levou em consideração o modelo teórico elaborado. Considerouse que os determinantes proximais da diarréia estavam situados no âmbito do controle de danos; os determinantes intermediários, situados no controle de riscos, e os determinantes distais, no controle de causas.

A atribuição de pontos para os critérios foi realizada da seguinte maneira: maior valor para aqueles critérios referentes ao controle de riscos (abastecimento de água, esgotamento sanitário, coleta de lixo, controle da qualidade da água e controle da qualidade de alimentos); valor intermediário para aqueles referentes ao controle de causas (educação sanitária); menor valor para os referentes ao controle de danos (qualidade do registro da informação nos prontuários e grau de adequação destes, articulação entre medidas curativas e preventivas, presença de barreiras geográficas, distribuição espacial das unidades de saúde, tempo de espera para marcar uma consulta, tempo de espera para ser atendido no consultório, porta de entrada no sistema e sistema de referência e contra-referência). Em tese, o controle de danos obteve menor peso, tendo em vista que a disponibilidade de atenção médica não tem relação causal com a ocorrência da diarréia; por outro lado, sua influência passa a ser determinante quando a doença já está instalada.

Em seguida, para cada critério, foram atribuídos pontos valendo-se da comparação entre a informação obtida nas diversas fontes e a imagem-objetivo delineada. Quando os pontos atribuídos a partir de fontes de dados distintas divergiam, era usado o valor médio. Posteriormente, o critério era classificado como incipiente, intermediário ou avançado, se obtivesse entre 0,00 e $33,30 \% ; 33,31 \%$ e $66,00 \% ; 66,10 \%$ e $100,00 \%$, respectivamente. Ao final, os escores foram tabulados em uma planilha do Excel (Figura 3) e o diagrama de síntese foi ali elaborado.

\section{Resultados}

\section{Acessibilidade}

A análise das trajetórias (Figura 2) revelou a existência de barreiras organizacionais, como os problemas relacionados à porta de entrada no sistema de serviços de saúde, visto que o primeiro atendimento, na maioria das vezes, era feito numa policlínica ou hospital públicos, mesmo existindo uma unidade básica de saúde próxima à casa das famílias.

Observou-se, também, que o agente comunitário de saúde não assumiu o papel de intermediário entre o domicílio da criança e uma unidade básica ou unidade de saúde da família, pois, no momento do adoecimento da criança, o agente não havia visitado a maioria dos domicílios estudados, exceto a casa de duas famílias.

O tempo de espera para o atendimento na unidade de saúde da família mostrou-se longo, se considerado em relação ao hospital ou policlínica. Observou-se, também, a presença de barreiras geográficas, visto que a distância per- 
Matriz de avaliação do controle da diarréia infantil.

\begin{tabular}{|c|c|c|c|c|}
\hline Dimensões & Pontos máximos & Sub-dimensões & Critérios & Pontos máximos \\
\hline Controle de causas & 30 & Ações de promoção à saúde & Educação sanitária & 30 \\
\hline \multirow[t]{5}{*}{ Controle de riscos } & 45 & Saneamento básico & Abastecimento de água & 20 \\
\hline & & & Esgotamento sanitário & 5 \\
\hline & & & Coleta de lixo & 5 \\
\hline & & Existência de ações de & Controle da água & 10 \\
\hline & & vigilância sanitária & Controle dos alimentos & 5 \\
\hline \multirow[t]{8}{*}{ Controle de danos } & 25 & Qualidade técnica do cuidado & Qualidade do registro da informação & 5 \\
\hline & & & Grau de adequação dos prontuários & 5 \\
\hline & & Integralidade do cuidado & Articulação entre medidas curativas & 5 \\
\hline & & $\begin{array}{l}\text { Uso de protocolo de atenção } \\
\text { à diarréia }\end{array}$ & e preventivas & 5 \\
\hline & & Acesso aos serviços de saúde & Presença de barreiras geográficas & 1 \\
\hline & & & $\begin{array}{l}\text { Distribuição espacial das unidades } \\
\text { de saúde }\end{array}$ & 1 \\
\hline & & & Tempo de espera para marcar consulta & 1,5 \\
\hline & & & Tempo de espera para ser atendido & 1,5 \\
\hline $\begin{array}{l}\text { Nível } 1 \text { - Práticas } \\
\text { assistenciais }\end{array}$ & 100 & & & 100 \\
\hline \multirow[t]{2}{*}{ Controle de danos } & 25 & Continuidade da assistência & $\begin{array}{l}\text { Porta de entrada no sistema de } \\
\text { serviços de saúde }\end{array}$ & 17,5 \\
\hline & & & $\begin{array}{l}\text { Sistema de referência e contra- } \\
\text { referência }\end{array}$ & 7,5 \\
\hline $\begin{array}{l}\text { Nível } 2 \text { - Coordenação } \\
\text { das práticas }\end{array}$ & 25 & & & 25 \\
\hline Total geral & 125 & & & 125 \\
\hline
\end{tabular}

corrida da casa da criança ao serviço de saúde pretendido requeria da maioria das mães alguma condução, e o percurso mais distante variou entre quarenta minutos de ambulância a uma hora a pé. Entretanto, associadas a isso, evidenciaram-se, também, barreiras financeiras, na medida em que as mães não possuíam dinheiro para pagar o transporte. Tal fato retardava o atendimento, e uma criança chegou a falecer em casa por esse motivo.

\section{Qualidade técnico-científica do cuidado}

A qualidade técnico-científica do cuidado foi considerada como inadequada, tendo em vista que não foi encontrado nenhum prontuário hospitalar ou ambulatorial completamente adequado para todos os itens - anamnese, exame físico, diagnóstico, tratamento e conclusão do caso. Entre os dez prontuários analisados, seis eram referentes ao atendimento ambulatorial e quatro, ao atendimento hospitalar. O número de prontuários hospitalares foi reduzido porque somente metade deles retornou com a análise concluída, pelo painel de especialistas, em tempo hábil.

Os prontuários hospitalares revelaram uma qualidade do cuidado um pouco melhor que aquela encontrada na rede básica. A anamnese foi considerada o item mais crítico para a atenção hospitalar, com apenas um prontuário adequado, seguida do exame físico e tratamento, ambos com dois prontuários adequados; na atenção ambulatorial, os itens que apresentaram as maiores falhas foram exame físico, considerado inadequado em seis prontuários analisados, tratamento e anamnese, ambos com apenas um prontuário adequado.

Outro ponto relevante foi a ausência de intervenção correta em um dos casos de interna- 
Figura 3

Grau de implantação das ações de controle da diarréia em município selecionado do Estado da Bahia,

Brasil, segundo as práticas assistenciais e coordenação das práticas, 2001.

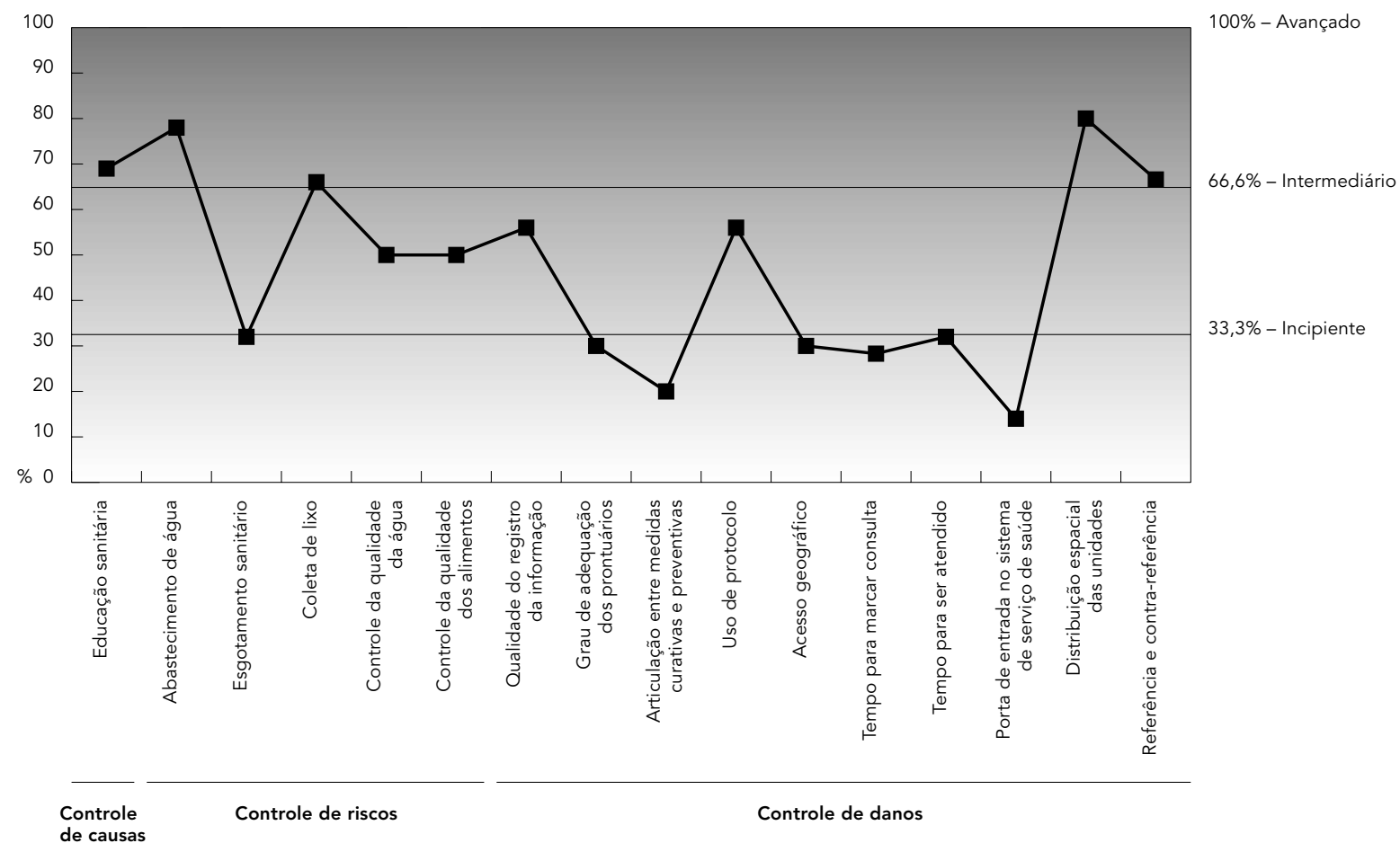

ção. Um dos membros do painel de especialistas verificou um longo espaço de tempo para o médico reavaliar uma criança em estado grave e o não encaminhamento desta para uma unidade de tratamento adequada, como unidade de terapia intensiva ou semi-intensiva, o que, possivelmente, ocasionou o óbito da criança.

Também a análise do tratamento instituído no hospital revelou falhas no manejo de dois casos, visto que uma das crianças fez uso indevido de antibioticoterapia, antiparasitários e reposição de volume, além de ter sido hospitalizada desnecessariamente. A outra não realizou alguns exames necessários, como hemogasimetria, dosagem de eletrólitos, hemocultura e urocultura, que serviriam para definir, adequadamente, o seu tratamento.

Entre os (seis) prontuários ambulatoriais, metade estava completamente inadequada, enquanto a outra metade apresentava apenas algum grau de adequação para um dos itens analisados isoladamente. Não se encontrou nenhum prontuário hospitalar completamente inadequado.

Em relação à qualidade do registro da informação, foi considerado regular tanto para o hos- pital, quanto para as unidades básicas, havendo registros ilegíveis e incompletos na maioria deles, inclusive naqueles preenchidos por profissionais que trabalham no Programa Saúde da Família (PSF). No hospital, em grande parte dos prontuários analisados, também se verificou a ausência de assinatura e carimbo dos profissionais que prestaram o atendimento.

\section{Grau de implantação das ações de controle da diarréia}

O município totalizou 66,2 pontos $(53,0 \%)$ de uma pontuação máxima possível de 125 pontos e obteve classificação intermediária em relação à imagem-objetivo proposta para avaliar a situação das ações implantadas para o controle da diarréia no nível local.

Quando se analisou separadamente cada nível, o desempenho do município foi melhor nas práticas assistenciais, com grau de implantação correspondente a 58,8\%, do que na coordenação, que atingiu 29,6\%. Portanto, o município foi classificado como intermediário no nível das práticas e incipiente na coordenação. 
Em relação às práticas assistenciais, a dimensão controle de causas obteve o melhor grau de implantação, com uma média de 20,7 pontos, perfazendo $69,0 \%$. Ao analisar o critério educação sanitária, observou-se que todos os agentes comunitários de saúde têm conhecimento acerca das medidas preventivas, porém uma parte deles não orienta as mães sobre o cuidado dispensado à criança com diarréia, apenas pesa as crianças, indica a necessidade de marcar uma consulta com o pediatra e mensura a pressão de adultos em suas visitas. Apenas alguns deles fazem orientações gerais sobre a higiene das crianças, preparo dos alimentos e tratamento adequado da água para o consumo.

A análise da orientação domiciliar, quando esta é realizada pelos agentes comunitários de saúde, revelou que eles sabem o que fazer em caso de diarréia, no que diz respeito tanto às medidas preventivas, quanto ao encaminhamento aos serviços de saúde. Apesar de todos os agentes terem feito curso abordando o tema diarréia, a maioria deles não soube descrever a forma de preparo dos sais de reidratação oral (SRO) e do soro caseiro, confundindo, inclusive, a maneira de fazer de um com a do outro. Apenas dois agentes o fizeram corretamente para apenas um dos soros.

Nas atividades educativas, existe pouca participação dos profissionais de nível superior, principalmente dos médicos, que permanecem praticando apenas a orientação individual em consultório, ainda que sejam profissionais inseridos no PSF. Quanto aos agentes, todos reconheceram os problemas de saneamento básico da área em que atuam, podendo ter contribuído para isso o seu longo período de residência nos bairros onde trabalham.

A dimensão controle de riscos obteve a segunda melhor pontuação, perfazendo um total de 28 pontos (62,0\%). Em relação ao saneamento básico, foram analisados o abastecimento de água, o esgotamento sanitário e a coleta de lixo. Desses critérios, o que apresentou alta pontuação foi o abastecimento de água, com 15,6 pontos $(78,0 \%)$, sendo considerado um critério avançado. A coleta de lixo foi classificada como intermediária, com 3,3 pontos $(66,0 \%)$, e o esgotamento sanitário foi o que apresentou a pior pontuação, com 1,6 pontos $(32,0 \%)$ e uma classificação incipiente.

Observou-se que a maioria dos domicílios recebe água encanada, mas quase todas têm apenas um ponto de água na porta de casa. É possível que a existência de um ponto de água externo imponha a manipulação da água e, conseqüentemente, a sua contaminação. Existe coleta regular de lixo, porém, em algumas áreas, a população o joga em lixões e, posteriormente, é feita a coleta. O esgotamento sanitário foi a área que se apresentou mais problemática, porque, apesar das iniciativas do gestor local em subsidiar fossa seca para algumas famílias, estas escoam os esgotos para a rua, para o fundo do próprio quintal ou jogam em um rio.

Os critérios referentes às ações de vigilância sanitária apresentaram classificação intermediária, com 50,0\% das ações implantadas, para cada um deles. Apesar de verificar, pela análise de documentos oficiais (Relatório da Vigilância Sanitária e Ambiental, janeiro a outubro de 2000), prioridade no controle da qualidade da água em comparação ao controle da qualidade dos alimentos, essa atividade só foi verificada nas centrais de distribuição de água do município e em estabelecimentos como unidades de saúde, creches e escolas. Observou-se que não é realizado o controle com amostras de água de domicílios em áreas de risco, como é o caso dos domicílios estudados. Entre as amostras analisadas, a causa predominante de contaminação foi a presença de coliformes fecais, entretanto, nesse documento, não são descritas as medidas adotadas pela vigilância sanitária para o tratamento desses reservatórios, nem são feitas referências ao acompanhamento de estabelecimentos comerciais visando a monitorizar a regularização da situação, após a notificação de alguma infração.

A dimensão controle de danos foi a que apresentou o menor escore, com 10,1 pontos obtidos (40,4\%), apesar de ter alcançado uma classificação intermediária quanto ao grau de implantação dessa prática. Dentre as suas subdimensões, a integralidade do cuidado foi a de menor desempenho, com $(20,0 \%)$, ao contrário das demais, como a distribuição espacial das unidades de saúde, com $(80,0 \%)$; o uso de protocolo de atenção à diarréia e a qualidade do registro da informação, ambos com (56,0\%), foram os que obtiveram a melhor pontuação. No que tange à integralidade do cuidado, observouse supremacia da abordagem curativa em relação às medidas preventivas e de promoção à saúde para o controle da diarréia. Dois profissionais entrevistados relatam adoção de conduta inadequada para tratar a diarréia, como introdução de antibiótico e suspensão da alimentação da criança, e os demais enfatizam a utilização dos planos A, B ou C para reidratar a criança. Nenhum deles participa de atividades educativas na comunidade onde trabalham, e apenas dois referiram já ter participado alguma vez dessas palestras em espaços comunitários.

As orientações fornecidas ao usuário, no momento da consulta, revelam a utilização de 
uma comunicação deficiente. Algumas mães relataram que, embora o médico tivesse lhes dado a receita, referindo que a criança precisava daquela medicação por causa do diagnóstico feito, não explicaram, com maiores detalhes, sua utilidade. Outras mães revelaram que não sabiam preparar os sais de reidratação oral e nem sabiam para que serviam, apesar de os terem oferecido a seu filho por orientação médica. Uma outra parte delas, apesar de conhecer o soro caseiro ou SRO, não o ofereceu a seu filho quando este estava com diarréia. Ou seja, os profissionais analisados pouco utilizam as oportunidades de consulta para discutir com a comunidade medidas preventivas para o controle da diarréia, ou para dar orientações gerais sobre estilo de vida e os riscos aos quais a família poderia estar exposta no momento da consulta; se o fizeram, a abordagem utilizada não teve eficácia a ponto de modificar o comportamento das mães. Apenas orientam, superficialmente, como tratar a doença. Em algumas falas dos agentes de saúde, pode-se perceber a baixa adesão ao tratamento prescrito e recidivas do paciente em relação ao mesmo problema. Além disso, encontrou-se uma baixa escolaridade e nível sócio-econômico das mães, o que pode estar funcionando como agravante.

Em relação à subdimensão acesso aos serviços de saúde, todos os critérios foram classificados como insuficientemente implantados, principalmente porque os pacientes tiveram dificuldade de ser atendidos na rede básica, exceto no que se refere à distribuição espacial das unidades de saúde. O tempo de espera para marcar uma consulta e para o atendimento no consultório só foi satisfatório para as unidades especializadas, como a policlínica ou o hospital, visto que esses serviços estão preparados para atender os casos de urgência e emergência.

Paradoxalmente, os maiores obstáculos foram relatados com referência à Unidade de Saúde da Família. Nestas, só existe atendimento à população adscrita, o qual é lento e por meio de distribuição de fichas. Em alguns casos, o número de fichas é insuficiente para atender à demanda, mesmo das famílias cadastradas, não havendo atendimento extra, inclusive nas situações de gravidade, segundo relato de uma das mães.

A distribuição geográfica dos serviços de saúde foi considerada como adequada, com 0,8 pontos $(80,0 \%)$, tendo sido classificada como avançada. Verificou-se um aumento do número de unidades de saúde e uma preocupação em direcionar as ações de saúde de acordo com o perfil epidemiológico de cada área. Observouse a existência de barreiras geográficas, princi- palmente, no que diz respeito à distância percorrida entre o domicílio da criança e a unidade de saúde, apresentando escore de 0,3 pontos $(30,0 \%)$.

Já a análise da utilização de protocolos de atenção à diarréia revelou que uma parte dos profissionais tinha o conhecimento de, pelo menos, um dos protocolos preconizados pelo Ministério da Saúde e o utilizava para o tratamento da diarréia, mas apenas um referiu conhecer e ter feito o curso AIDPI, e dois dos profissionais entrevistados não conheciam nem utilizavam protocolos, o que correspondeu a um escore de 2,8 pontos $(56,0 \%)$.

Em relação à coordenação das práticas, um dos critérios investigados foi a porta de entrada no sistema de saúde, que se revelou inadequada. Os usuários, na maioria dos casos estudados, recorreram ao hospital ou às policlínicas referindo problemas no acesso à rede básica, o que implicou uma pontuação baixa, de 2,4 pontos (14,0\%), e uma classificação incipiente.

Já o sistema de referência e contra-referência funciona parcialmente (cinco pontos, 66,6\%). A referência da rede hospitalar para a rede básica funciona, visto que todos os casos que passaram pelo hospital foram encaminhados para uma unidade básica de saúde; também se observou a utilização da ficha de referência padrão para fazer o encaminhamento dos pacientes. Há, contudo, problemas na referência entre a rede básica e a especializada. Poucos prontuários ambulatoriais apresentavam conclusão da conduta e ficha de referência para unidades de maior complexidade, quando se fazia necessário. Vale ressaltar que as mães não procuraram essas unidades após alta hospitalar porque, na percepção delas, seus filhos já estavam curados.

\section{Discussão}

A presente investigação revelou que, para os casos de óbitos e hospitalizações investigados, algumas ações de controle à diarréia estavam insuficientemente implantadas no município, no ano de 2001. As medidas de controle adotadas apresentaram falhas e lacunas que justificaram a existência de hospitalizações e óbitos evitáveis naquele período. Embora o abastecimento de água tenha sido um dos poucos critérios que atingiu pontuação elevada, há contaminação da água em locais estratégicos, como escolas, creches e unidades de saúde, revelando que essa medida ainda não garante a cobertura requerida para proteger a população. Constatou-se que a população tem buscado 
centros de saúde distantes do seu local de residência em virtude, principalmente, da presença de barreiras organizacionais nas unidades de saúde, mesmo havendo boa distribuição espacial destas.

Observou-se que o acesso às unidades de atenção primária, como as Unidades de Saúde da Família, foi prejudicado por causa da nãoflexibilização da rotina para atender os pacientes quando as fichas de atendimento acabavam. Por essa razão, os pacientes buscaram, nos casos que foram a óbito e/ou tiveram que ser internados por diarréia, como porta de entrada no sistema de saúde, os serviços de atenção especializada, como policlínica e hospital públicos. A ausência de um ponto de entrada facilmente acessível faz com que a atenção adequada possa não ser obtida ou seja adiada, incorrendo em gastos adicionais 16 .

A insuficiência do registro das informações verificada nos prontuários, em toda a rede de serviços, foi concordante com os achados de outros estudos 19,27. Pereira 19 relaciona essa deficiência à pressa para anotar as informações ou ao fato de o médico não acreditar na utilidade desses registros.

Outro aspecto que vale destacar diz respeito à baixa qualidade técnica do cuidado. Supostamente, isso poderia estar relacionado ao despreparo dos profissionais para atuar dentro dos parâmetros dos programas de assistência integral do Ministério da Saúde, como também ao desconhecimento ou à pouca utilização de protocolos para atendimento a agravos específicos. Formigli et al. 27 já haviam observado, em seu estudo, que a qualidade do atendimento prestado às crianças menores de um ano que foram a óbito, em Salvador, no ano de 1991, estava abaixo dos padrões desejáveis, tendo em vista o conhecimento e a tecnologia disponíveis.

Acredita-se que todos os óbitos investigados poderiam ter sido evitados se as crianças tivessem tido, também, uma intervenção precoce dos agentes comunitários de saúde. Esses profissionais não desenvolveram ações educativas com a família quando do adoecimento da criança. A maioria deles sequer sabia preparar corretamente o soro caseiro ou os sais de reidratação oral, não tendo indicado, devidamente, o uso dos mesmos. Observou-se que, em nenhum dos casos, a cobertura da área pelo Programa de Agentes Comunitários de Saúde mostrou-se como um diferencial para a cura das crianças. Entre as possíveis causas para o desconhecimento dos agentes comunitários de saúde quanto ao método para o preparo do soro, pode-se identificar a utilização de abordagens pedagógicas insuficientes ou inade- quadas nos treinamentos oferecidos a esses profissionais.

Verificou-se, ainda, que, em nenhum dos casos estudados, os pacientes seguiram a orientação de procurar uma unidade básica de saúde para acompanhamento ambulatorial, fato que impossibilitou avaliar a continuidade da assistência.

Os achados deste estudo são complementares àqueles de investigações de caráter mais extensivo, como a análise de séries temporais 7, que têm mostrado a persistência da mortalidade infantil evitável. Por meio dele, foi possível reunir informações mais detalhadas acerca das possíveis causas para os óbitos infantis nas dimensões do acesso, qualidade técnica e integralidade do cuidado. Todavia, dentre as limitações desta pesquisa, está a atribuição de pesos na matriz de análise das ações de controle da diarréia, pelo fato de ter sido feita com base no referencial teórico. Tendo em vista que toda atribuição de pesos envolve certa arbitrariedade, submetê-la a um painel de especialistas teria resultado em sua maior validade.

Cabe, ainda, um comentário a respeito da perda da informação sobre metade dos prontuários hospitalares que não foram analisados, o que comprometeu a representatividade acerca da qualidade do cuidado no hospital.

\section{Considerações finais}

A ocorrência de hospitalizações e óbitos infantis por diarréia, evitáveis por meio de tecnologia simples e amplamente difundida, parece relacionar-se à insuficiência da implantação das ações de controle a esse agravo.

A estratégia metodológica adotada no presente estudo permitiu a identificação de vários problemas relacionados ao acesso, à qualidade e à continuidade do cuidado que estudos mais extensivos não revelam. Os instrumentos aqui desenvolvidos possibilitaram avaliar, com maior profundidade, as práticas assistenciais e a qualidade da atenção no nível local. Podem ser simplificados e adaptados para avaliação rotineira dos serviços de saúde.

Alguns dos achados desta investigação requerem, contudo, a realização de investigações adicionais voltadas para esclarecer algumas questões que fugiam aos objetivos deste trabalho, tais como as razões da baixa qualidade técnico-científica do cuidado em instituições públicas e a falta de efetividade de certas estratégias pedagógicas para o treinamento de agentes comunitários de saúde. 


\section{Resumo}

Com o objetivo de avaliar a qualidade técnico-científica do cuidado, a acessibilidade aos serviços de saúde e o grau de implantação das ações de controle da diarréia, em um município selecionado do Estado da Bahia, Brasil, realizou-se um estudo de casos múltiplos, com dois níveis de análise: as práticas e a coordenação destas. Cada óbito (três) e hospitalização (oito) por diarréia foram considerados como casos. A totalidade dos óbitos foi investigada e as hospitalizações foram selecionadas de forma intencional. Dimensões e critérios para a avaliação da qualidade técnica e para estimativa do grau de implantação das ações de controle foram derivadas do modelo teórico. As trajetórias assistenciais foram reconstituídas a partir de entrevistas e informações de prontuários médicos. Verificaram-se barreiras organizacionais relacionadas ao elevado tempo de espera para atendimento nas unidades de atenção primária; barreiras geográficas relacionadas à distância entre o serviço e o local de moradia dos usuários e barreiras financeiras decorrentes da escassez de recursos financeiros para o transporte. A qualidade do cuidado foi considerada inadequada e as ações de controle da diarréia foram classificadas como insuficientes em praticamente todas as dimensões analisadas.

Avaliação dos Serviços de Saúde; Diarréia; Acesso aos Cuidados de Saúde; Qualidade do Cuidado de Saúde

\section{Colaboradores}

R. M. Lopes contribuiu com a definição da pergunta e do desenho, fez a revisão de literatura, executou o trabalho de campo, realizou a análise dos dados e a redação do trabalho. L. M. Vieira-da-Silva concebeu a arquitetura geral do estudo e o desenho da pesquisa, auxiliou na análise dos dados e na correção e redação do texto; Z. M. A. Hartz detalhou as dimensões e categorias de análise da matriz de análise do controle da diarréia, contribuiu na análise das trajetórias.

\section{Agradecimentos}

Ao apoio financeiro dado pelo Ministério da Saúde/ Fundação de Amparo à Pesquisa do Estado da Bahia para o desenvolvimento deste estudo.

\section{Referências}

1. Guimarães ZA, Costa, MC, Paim JS, Silva LM. Declínio e desigualdades sociais na mortalidade infantil por diarréia. Rev Soc Bras Med Trop 2001; 34:473-8.

2. Fundo das Nações Unidas para a Infância. Situação mundial da infância. Brasília: Fundo das Nações Unidas para a Infância; 2000.

3. Ministério da Saúde. Atenção integrada às doenças prevalentes na infância. 2a Ed. Brasília: Ministério da Saúde; 2002.

4. Szwarcwald CL, Leal MC, Castilho EA, Andrade CLT. Mortalidade infantil no Brasil: Belíndia ou Bulgária? Cad Saúde Pública 1997; 13:503-16.

5. Oliveira LAP, Mendes MMS. Mortalidade infantil no Brasil: uma avaliação de tendências recentes. In: Minayo MCS, organizador. Os muitos brasis: saúde e população na década de 80 . Rio de Janeiro: Editora Hucitec; 1995. p. 291-301.

6. Costa MCO. Determinantes da variação da mortalidade infantil no Brasil: a contribuição da fecundidade e de variáveis sócio-ambientais [Tese de Doutorado]. Salvador: Instituto de Saúde Coletiva, Universidade Federal da Bahia; 2001.

7. Oliveira AB. Mortalidade infantil por causas evitáveis em Salvador, 2001 [Dissertação de Mestrado]. Salvador: Instituto de Saúde Coletiva, Universidade Federal da Bahia; 2001.

8. Kosek M, Bern C, Guerrant RL. The global burden of diarrhoeal disease, as estimated from studies published between 1992 and 2000. Bull World Health Organ 2003; 81:197-204.

9. Hartz ZMA, Champgne F, Leal MC. Mortalidade infantil "evitável" em duas cidades do Nordeste do Brasil: indicador de qualidade do sistema local de saúde. Rev Saúde Pública 1996; 34:310-8.

10. Paim JS. A reforma sanitária e os modelos assistenciais. In: Rouquayrol MZ, organizador. Epidemiologia \& saúde. 4a Ed. Rio de Janeiro: Editora Medsi; 1994. p. 455-66.

11. Paim JS. A reforma sanitária e os modelos assistenciais. In: Rouquayrol MZ, organizador. Epidemiologia \& saúde. 5a Ed. Rio de Janeiro: Editora Medsi; 1999. p. 473-87.

12. Paim JS. Modelos assistenciais: reformulando o pensamento e incorporando a proteção e a promoção da saúde. In: Paim JS, organizador. Saúde, política e reforma sanitária. Salvador: Instituto de Saúde Coletiva/Centro de Estudos e Projetos em Saúde; 2002. p. 367-81.

13. Kessner DM, Kalk CE, Singer J. Assessing health quality. The case for tracers. N Engl J Med 1973; 288:189-94.

14. Rutstein DD, Berenberg W, Chalmers TC, Child CG, Fishman AP, Perrin EB. Measuring the quality of medical care: a clinical method. N Engl J Med 1976; 294:582-8.

15. Frenk J. El concepto y la medición de accesibilidad. Salud Publica Mex 1985; 27:438-53.

16. Starfield B. Responsabilidade na atenção primária. In: Ministério da Saúde, organizador. Atenção primária - equilíbrio entre necessidades de saúde, serviços e tecnologia. Brasília: Organização das Nações Unidas para a Educação, a Ciência e a Cultura/Ministério da Saúde; 2002. p. 207-45. 
17. Vuori H. A qualidade da saúde. Saúde para debate. Rio de Janeiro: Centro Brasileiro de Estudos de Saúde; 1991.

18. Donabedian A. Evaluación de la calidad de la atención médica. In: White KL, editor. Investigaciones sobre servicios de salud: una antología. Washington DC: Organización Panamericana de la Salud; 1992. p. 382-404. (Publicacion Científica 534).

19. Pereira MG. Qualidade dos serviços de saúde. In: Pereira MG, organizador. Epidemiologia - teoria e prática. Rio de Janeiro: Editora Guanabara Koogan. 1995. p. 538-60.

20. Giovanella L, Lobato LVC, Carvalho AI, Conill EM, Cunha EM. Sistemas municipais de saúde e a diretriz da integralidade da atenção: critérios para avaliação. Saúde Debate 2002; 26:37-61.

21. Lopes RM. Avaliação da atenção à diarréia na infância: organização, acesso e qualidade técnica dos serviços [Dissertação de Mestrado]. Salvador: Instituto de Saúde Coletiva, Universidade Federal da Bahia; 2004.

22. Organização Mundial da Saúde. Manual da classificação estatística internacional de doenças, lesões e causas de óbitos - nona conferência de revisão. São Paulo: Centro Brasileiro de Classificação de Doenças em Português; 1985.
23. Organização Mundial da Saúde. Classificação estatística internacional de doenças e problemas relacionados à saúde, 10a Revisão. v. 1. São Paulo: Centro Colaborador da OMS para a Classificação de Doenças em Português; 1995.

24. Araújo MD. Avaliação da qualidade da atenção hospitalar às pneumonias na infância [Dissertação de Mestrado]. Salvador: Instituto de Saúde Coletiva, Universidade Federal da Bahia; 1996.

25. Kennedy HP. Enhancing Delphi Research: methods and results. J Adv Nurs 2004; 45:504-11.

26. Starfield B. Coordenação da atenção - juntando tudo. In: Ministério da Saúde, organizador. Atenção primária - equilíbrio entre necessidades de saúde, serviços e tecnologia. Brasília: Organização das Nações Unidas para a Educação, a Ciência e a Cultura/Ministério da Saúde. 2002. p. 365-414.

27. Formigli VL, Vieira-da-Silva LM, Cerdeira AJP, Pinto CME, Oliveira RSA, Caldas AC, et al. Avaliação da atenção à saúde através da investigação de óbitos infantis. Cad Saúde Pública 1996; 12 Suppl 2:33-41.

Recebido em 21/Jan/2004

Versão final reapresentada em 15/Jul/2004

Aprovado em 29/Jul/2004 